\title{
High Monocyte-To-Lymphocyte Ratio Is Associated With Stroke-Associated Pneumonia
}

\author{
Hao-Ran Cheng ${ }^{1 \dagger}$, Jia-Ying Song ${ }^{2 \dagger}$, Yi-Nuo Zhang ${ }^{1}$, Yun-Bin Chen ${ }^{1}$, Gang-Qiang Lin ${ }^{1}$, \\ Gui-Qian Huang ${ }^{1 *}$, Jin-Cai He ${ }^{1 *}$ and Zhen Wang ${ }^{1 *}$ \\ ${ }^{1}$ Department of Neurology, The First Affiliated Hospital of Wenzhou Medical University, Wenzhou, China, ${ }^{2}$ School of Mental \\ Health, Wenzhou Medical University, Wenzhou, China
}

OPEN ACCESS

Edited by:

Anna Rosell,

Vall d'Hebron Research Institute (NHIR), Spain

Reviewed by:

Steffen Tiedt,

Ludwig-Maximilians-Universität

München Munich University Hospital, Germany

Wen-Jun Tu,

Chinese Academy of Medical Sciences and Peking Union Medical

College, China

*Correspondence:

Zhen Wang

wangzhen_wenzhou@163.com

Jin-Cai He

hic@wmu.edu.cn

Gui-Qian Huang

huangguiqian123@126.com

†These authors have contributed equally to this work

Specialty section:

This article was submitted to Stroke,

a section of the journal

Frontiers in Neurology

Received: 24 June 2020

Accepted: 25 August 2020

Published: 06 October 2020

Citation:

Cheng $H-R$, Song $J-Y$, Zhang $Y-N$,

Chen $Y-B$, Lin $G-Q$, Huang $G-Q$,

He J-C and Wang Z (2020) High Monocyte-To-Lymphocyte Ratio is Associated With Stroke-Associated

Front. Neurol. 11:575809.

doi: 10.3389/fneur.2020.575809
Purpose: Stroke-associated pneumonia (SAP), a common complication in acute ischemic stroke (AIS) patients, is associated with poor prognosis after AIS. Inflammation plays an important role in the development of SAP. In this study, we aimed to explore the association between the monocyte-to-lymphocyte ratio (MLR) and SAP in AIS patients.

Methods: We continuously enrolled 972 AIS patients. SAP was diagnosed by two trained neurologists and confirmed by radiography, meeting the modified Centers for Disease Control and Prevention criteria. MLR values were measured for all participants, and all patients were evenly classified into three tertiles according to the MLR levels. We used the values that Youden's index max points corresponded to represent the optimal cutoffs, which represented the balance in sensitivity and specificity.

Results: $104(10.7 \%)$ patients were diagnosed with SAP. SAP patients showed a significant increased $(P<0.001)$ MLR when compared with non-SAP. The optimal cutoff points of MLR were (T1) < 0.2513, (T2) 0.2513-0.3843, and (T3) $>0.3843$. The incidence of SAP was significantly higher in the third MLR tertile than the first and second MLR tertiles (21.7 vs. 4 vs. $6.5 \%$, respectively, $P<0.001$ ). After adjusting for confounding and risk factors, multivariate regression analysis showed that the third MLR tertile was an independent variable predicting the occurrence of SAP (odds ratio $=3.503$, $95 \% \mathrm{Cl}=1.066-11.515, P=0.039$ ).

Conclusions: Our study showed that higher MLR was significantly associated with SAP in AIS patients. MLR is beneficial for clinicians to recognize patients with a high risk of SAP at an early stage and is an effective way to improve clinical care of SAP patients. Higher MLR could be a helpful and valid biomarker for predicting SAP in clinical practice.

Keywords: acute ischemic stroke, stroke-associated pneumonia, inflammation, monocyte, lymphocyte

\section{INTRODUCTION}

Stroke-associated pneumonia (SAP) is one of the most common complications after acute ischemic stroke (AIS), with an incidence of 6.7-36.98\% (1-3), and may lead to lengthy hospitalization, poor functional outcome, and high morbidity and mortality $(2,4-7)$. It has been previously confirmed that the use of prophylactic antibiotics does not prevent SAP $(8,9)$. Due to the extra clinical and financial burden associated with SAP, it is necessary to explore underlying risk factors to aid with early recognition and prevention. 
The SAP-related poor prognosis and several risk factors have been recognized in previous studies, creating several predictive models of SAP (5, 10-14). Hoffmann et al. developed and validated the $\mathrm{A}^{2} \mathrm{DS}^{2}$, a 10 -point clinical model with high sensitivity and specificity for predicting $\operatorname{SAP}(3,14,15)$. This model assesses risk factors, including age, atrial fibrillation, dysphagia, sex, and previous stroke severity. In a total of 3,160 Chinese AIS patients, Li et al. (16) used machine learning methods to develop a model with high sensitivity and specificity to predict SAP. Interestingly, a study found that reduced vitamin D was a potential risk factor of SAP (17). In 2019, ZapataArriaza et al. (18) found that soluble urokinase plasminogen activator receptor and serum amyloid $\mathrm{A}$, which were determined by immunoassays, were promising tools in early diagnosis of SAP. Advanced age, male, stroke severity, dysphagia, and low estimated glomerular filtration rate (eGFR) are predictive factors for $\operatorname{SAP}(5,12,13)$. In an animal experiment, C57BL/6 mice treated with an anti-CD147 antibody could decrease the lung damages, bacterial load, and pulmonary edema after receiving middle cerebral artery occlusion (19).

Evidence has found that inflammation is important in the development of SAP, and the associations between SAP and inflammatory biomarkers, such as interleukin 6, neutrophil-tolymphocyte ratio, and C-reactive protein, have been explored $(20,21)$. Monocyte-to-lymphocyte ratio (MLR) is the absolute monocyte count divided by the absolute lymphocyte count and has been demonstrated to be a novel hematological and inflammatory parameter. MLR is associated with various diseases, such as community-acquired pneumonia, axial spondylarthritis, and coronary angiography, as well as the systemic inflammatory response, which reflects the abnormal immune status of diseases (22-24); however, the relationship between SAP and MLR remains unclear. In 2017, a study found that lymphocyte-to-monocyte ratios (LMRs) at admission were lower in AIS patients with pneumonia or urinary tract infection compared with patients without infections (25). Alternatively, the platelet-to-lymphocyte ratio (PLR) is the absolute platelet count divided by the absolute lymphocyte count, is a biomarker of systemic inflammation, and is related to the prognosis of hepatocellular carcinoma and the cognitive functions of breast cancer survivors $(26,27)$. Furthermore, leukocytes, monocytes, and lymphocytes have different roles in the inflammatory process, and their counts could directly reflect the inflammatory process. Meanwhile, the relationship between plasma biomarkers and AIS has been studied in many areas of stroke researches and found that biomarkers may help a lot in the early stage of AIS. Tu et al. (28) found that copeptin, as a plasma neuroendocrine biomarker, showed great ability in predicting a 3 month functional outcome and mortality after AIS.

To date, researches concerning the relationships between SAP and easy obtained blood biomarkers and comparing the predictive values of different biomarkers were insufficient. Our study could fill these gaps in previous studies and find the economical, objective, simple blood biomarkers. Thus, these biomarkers could help clinicians recognize AIS patients with a high risk of SAP at an early stage as well as reduce the financial and caring burden of patients. The purpose of the present study was to investigate the association between MLR and SAP in AIS patients, as well as find a helpful and valid biomarker for predicting and evaluating SAP in clinical practice.

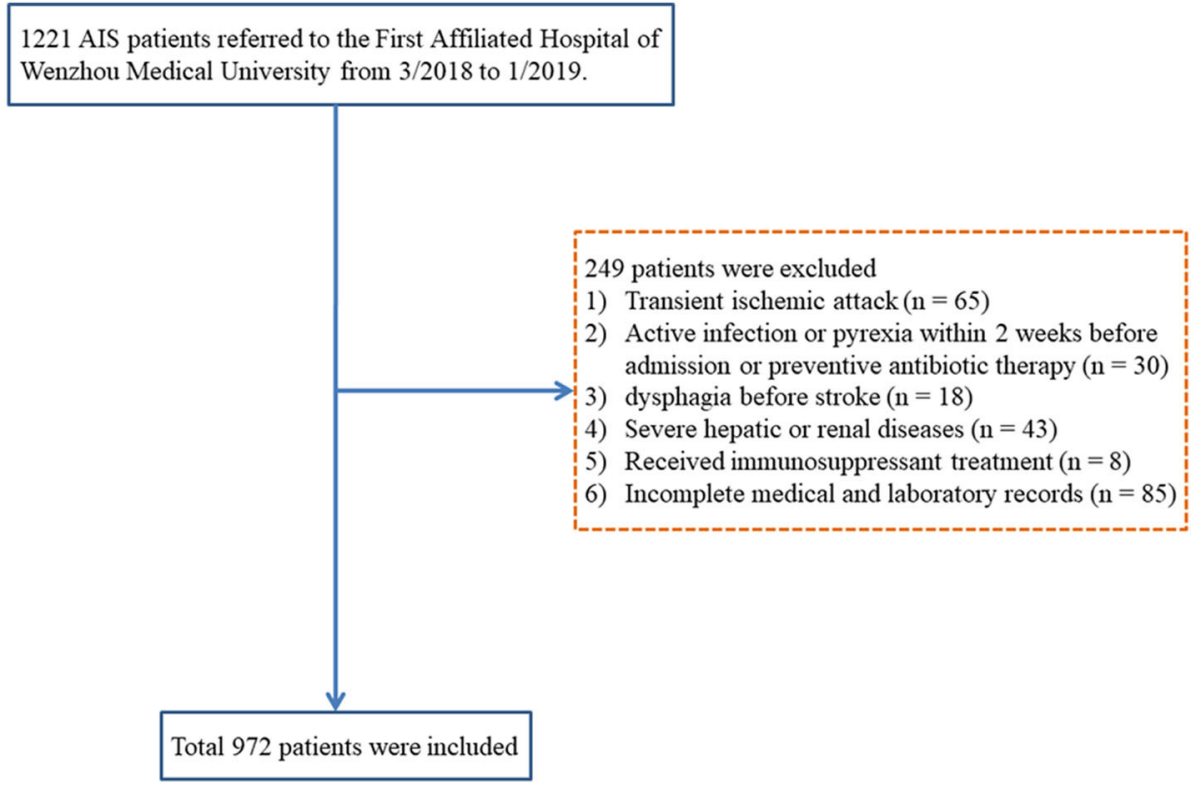

FIGURE 1 | Research flowchart. AIS, acute ischemic stroke. 


\section{MATERIALS AND METHODS}

\section{Subjects}

The present study was a retrospective study of patients with and without SAP. We obtained the approval of the Ethics Committee of the First Affiliated Hospital of Wenzhou Medical University (NO. 2019042) and conducted the study at the Neurology Department of the First Affiliated Hospital of Wenzhou Medical University under the Declaration of Helsinki.

Patients, who were diagnosed with AIS and hospitalized in the Neurology Department of the First Affiliated Hospital of Wenzhou Medical University from March 2018 to January 2019, were continuously enrolled. The inclusion criteria were age $\geq 18$ years and diagnosis of AIS confirmed by computerized tomography (CT) or magnetic resonance imaging at the time of admission. The exclusion criteria were transient ischemic attack; active infection or pyrexia within 2 weeks of admission or prophylactic antibacterial therapy; dysphagia before stroke; patients with any severe liver or kidney dysfunction; a history of the hematological disease, cancer, or received immunosuppressant treatment; pneumonia before stroke; or if patient's medical record was incomplete. A total of 972 AIS patients were enrolled in our present study (Figure 1).

\section{Diagnosis of Stroke-Associated Pneumonia}

The diagnosis of SAP was based on the modified Center for Disease Control and Prevention criteria. SAP was defined as the infection of the lower respiratory tract during the first 7 days

TABLE 1 | Baseline characteristics of AIS patients stratified by SAP.

\begin{tabular}{|c|c|c|c|c|}
\hline & All patients & Non-SAP $(n=868)$ & SAP $(n=104)$ & $P$-value \\
\hline Age (years), median (IQR) & $67.0(59.0-74.0)$ & $66.0(59.0-73.0)$ & $72.0(65.0-80.0)$ & $<0.001$ \\
\hline Male, $n(\%)$ & $619(63.7 \%)$ & $553(63.7 \%)$ & $66(63.5 \%)$ & 0.960 \\
\hline Drinking, $n(\%)$ & $361(37.1 \%)$ & $319(63.2 \%)$ & $42(40.4 \%)$ & 0.469 \\
\hline Baseline SBP (mmHg) & $152.76 \pm 24.34$ & $152.50 \pm 24.38$ & $154.96 \pm 23.94$ & 0.330 \\
\hline Previous stroke, $n(\%)$ & $141(14.5 \%)$ & $121(13.9 \%)$ & $20(19.2 \%)$ & 0.148 \\
\hline Hypertension, $n$ (\%) & $746(76.7 \%)$ & $665(76.6 \%)$ & $81(77.9 \%)$ & 0.095 \\
\hline Diabetes, $n(\%)$ & $390(40.1 \%)$ & $356(41.0 \%)$ & $34(32.7 \%)$ & 0.855 \\
\hline CAD, $n(\%)$ & $21(2.2 \%)$ & $21(2.4 \%)$ & $0(0.0 \%)$ & 0.155 \\
\hline $\mathrm{AF}, n(\%)$ & $118(12.1 \%)$ & $100(11.5 \%)$ & $18(17.3 \%)$ & 0.088 \\
\hline Use of nasogastric tubes, $n(\%)$ & 95 (9.8\%) & $78(9.0 \%)$ & $17(16.3 \%)$ & 0.017 \\
\hline $\mathrm{A}^{2} \mathrm{DS}^{2}$ score, median (IQR) & $1.0(1.0-3.0)$ & $1.0(1.0-3.0)$ & $3.0(1.0-5.0)$ & $<0.001$ \\
\hline RBC $\left(\times 10^{12} / \mathrm{L}\right)$, median (IQR) & $4.45(4.14-4.77)$ & $4.45(4.16-4.77)$ & $4.38(3.98-4.68)$ & 0.061 \\
\hline Leukocyte $\left(\times 10^{9} / \mathrm{L}\right)$, median (IQR) & $6.72(5.57-8.26)$ & $6.58(5.50-7.92)$ & $8.36(6.32-11.05)$ & $<0.001$ \\
\hline Neutrophils $\left(\times 10^{9} / \mathrm{L}\right)$, median (IQR) & $4.24(3.26-5.67)$ & $4.14(3.21-5.36)$ & $6.15(4.21-8.41)$ & $<0.001$ \\
\hline Monocyte (×109/L), median (IQR) & $0.51(0.39-0.67)$ & $0.50(0.38-0.64)$ & $0.68(0.45-1.00)$ & $<0.001$ \\
\hline Lymphocyte (×109/L), median (IQR) & $1.64(1.28-2.07)$ & $1.67(1.32-2.09)$ & $1.30(1.03-1.69)$ & $<0.001$ \\
\hline PLT (×109/L), median (IQR) & $219.00(182.00-257.50)$ & 219.00 (184.00-257.50) & $215.00(171.25-258.75)$ & 0.291 \\
\hline FBG (mmol/L), median (IQR) & $5.60(4.80-7.43)$ & $5.60(4.80-7.50)$ & $5.80(4.90-7.20)$ & 0.788 \\
\hline $\operatorname{Scr}(\mu \mathrm{mol} / \mathrm{L})$, median $(\mathrm{IQR})$ & $71.00(59.00-84.00)$ & $71.00(59.00-84.00)$ & $71.00(58.00-85.00)$ & 0.744 \\
\hline GFR, median (IQR) & $90.90(75.30-101.70)$ & $91.15(75.65-102.80)$ & $86.60(73.40-95.60)$ & 0.015 \\
\hline
\end{tabular}

SBP, systolic blood pressure; DBP, diastolic blood pressure; NIHSS, National Institute of Health Stroke Scale; CAD, coronary artery disease; AF, atrial fibrillation; PLT, platelet; FBG, fasting blood glucose; GFR, glomerular filtration rate; MLR, monocyte-to-lymphocyte ratio; PLR, platelet-to-lymphocyte ratio. 
of hospitalization after stroke onset $(20,29)$, based on clinical symptoms, laboratory measurements, and confirmation via chest X-ray and CT (30). This study documented only hospitalacquired pneumonia and community-acquired pneumonia, and pneumonia before the stroke was not taken into consideration. SAP diagnosis was performed by two trained neurologists who were blinded to the patient's data.

\section{Data Collection}

We collected demographic and clinical data retrospectively, including age, sex, history of previous disease, smoking, and drinking. Baseline blood pressure, thrombolytic therapy, stroke subtype (Trial of ORG 10172 in Acute Stroke Treatment criteria), and National Institutes of Health Stroke Scale (NIHSS) scores $24 \mathrm{~h}$ after admission were also recorded. Furthermore, all participants underwent the modified water swallowing test, a Japanese bedside dysphagia assessment, within $24 \mathrm{~h}$ after admission. In the modified water swallowing test, patients were asked to drink $30 \mathrm{~mL}$ water, and a trained neurologist would evaluate the swallowing reflex, wet hoarseness, and cough (31, 32). The existence of dysphagia, the use of mechanical ventilation, and nasogastric tubes were recorded to reflect swallowing

TABLE 2 | Baseline characteristics of AIS patients in different MLR tertiles.

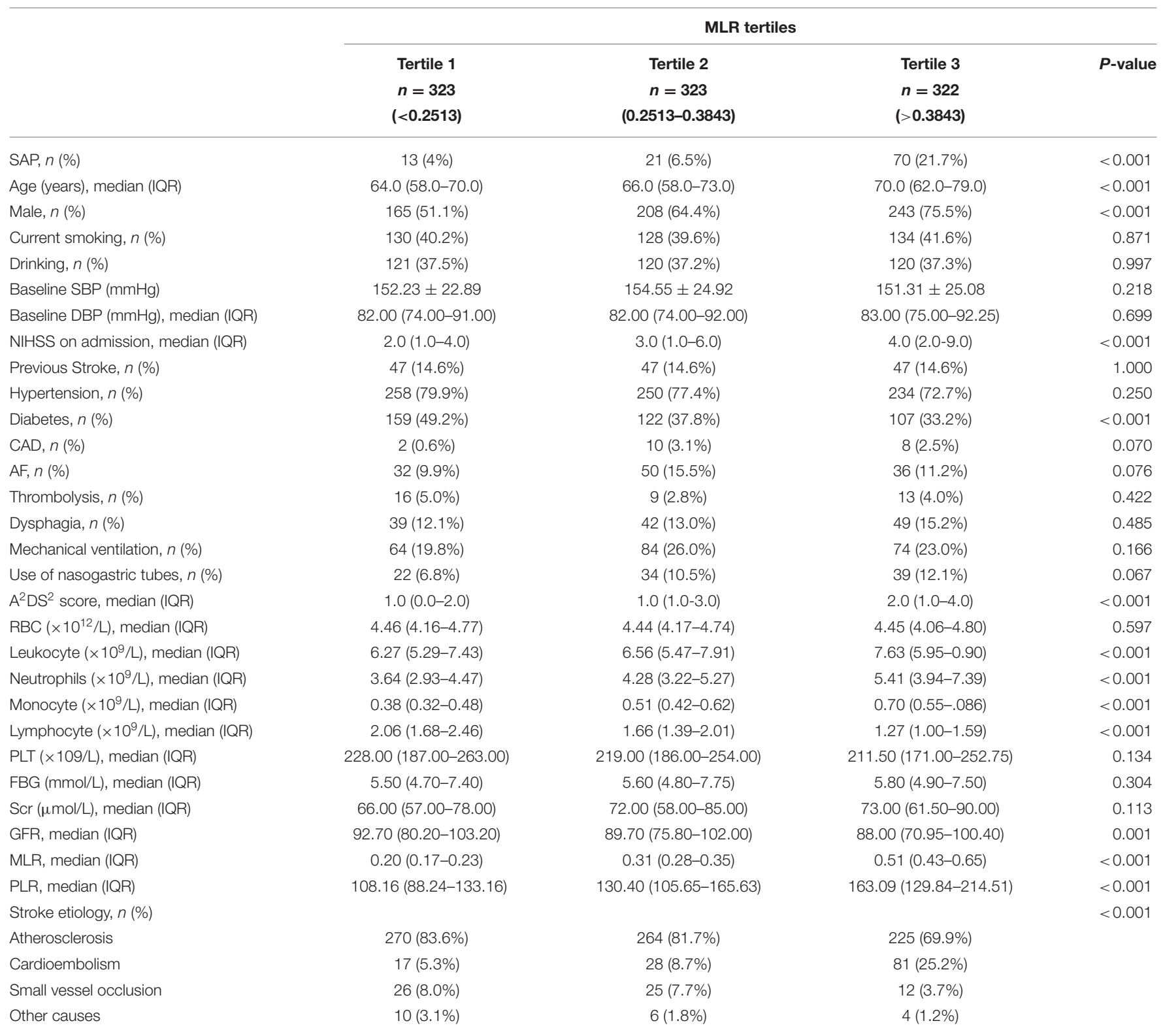

SBP, systolic blood pressure; DBP, diastolic blood pressure; NIHSS, National Institute of Health Stroke Scale; CAD, coronary artery disease; AF, atrial fibrillation; PLT, platelet; FBG, fasting blood glucose; GFR, glomerular filtration rate; MLR, monocyte-to-lymphocyte ratio; $P L R$, platelet-to-lymphocyte ratio. 
functions. $\mathrm{A}^{2} \mathrm{DS}^{2}$ score was also rated for subsequent combined analysis. This score adds up scores of each item including age ( $\geq 75$ years, 1 point), atrial fibrillation (yes, 1 point), dysphagia (yes, 2 points), sex (male, 1 point), and stroke severity (0-4 NIHSS score, 0 points; $5-15$ NIHSS score, 3 points; $\geq 16$ NIHSS score, 5 points).

Within $24 \mathrm{~h}$ of hospital admission, blood from the antecubital vein was drawn and immediately sent for analysis. Laboratory parameters included red blood cells, leukocytes, neutrophils, monocytes, lymphocytes, platelets, fasting blood glucose, serum creatinine, and estimated glomerular filtration rate. All patients were evenly classified into three tertiles according to the MLR levels (tertile 1, <0.2513; tertile 2, 0.2513-0.3843; and tertile $3,>0.3843)$.

\section{Statistical Analysis}

Continuous variables were analyzed using $t$-test or Mann-Whitney tests, presented as mean \pm standard deviation or medians (quartiles). Categorical variables were compared using chi-square or Fisher's exact tests and denoted as relative frequencies and percentages. The Kruskal-Wallis test or one-way analysis of variance were used to compare the difference between three MLR tertiles in continuous variables, whereas Pearson's chi-square or Fisher's exact tests were used in categorical variables. Multiple logistic regression was used to adjust for potential confounding or risk factors. Receiver operating characteristics (ROC) curve analysis was used to determine the significant cutoff value, sensitivity, and specificity. The optimal cutoffs were values that Youden's index max points corresponded, which made the diagnostic effectiveness reach the best objectively and represent the balance in sensitivity and specificity (33). MLR and other biomarker values that were used to predict SAP were analyzed and compared using the area under the ROC curve (AUROC). Z-test was used for comparing ROC curves of MLR and other biomarker values (34). All statistical analyses used SPSS (version 19.0.0; IBM, Armonk, NY, USA) and MedCalc (version 13.0; MedCalc Software Ltd., Ostend, Belgium). Two-tailed $P$-values $<0.05$ were considered statistically significant.

\section{RESULTS}

\section{Baseline Characteristics of Acute Ischemic Stroke Patients Stratified by Stroke-Associated Pneumonia}

During the research period, 972 AIS patients were included, and $104(10.7 \%)$ patients were diagnosed with SAP (Figure 1). The baseline demographic, clinical, and laboratory variables are displayed in Table 1. A total of 619 (63.7\%) patients were male, and the mean age of the enrolled patients was 67.0 years (59.0-74.0 years). Compared with non-SAP patients, SAP patients were older $(P<0.001)$, had higher NIHSS scores at admission $(P<0.001)$, were more likely to acquire dysphagia $(P=0.031)$, and were more likely to use nasogastric tubes $(P=0.017)$. For laboratory parameters, SAP patients had higher leukocyte counts, monocyte counts, MLR, and PLR, whereas
non-SAP patients had lower lymphocyte counts. Furthermore, SAP patients demonstrated higher $\mathrm{A}^{2} \mathrm{DS}^{2}$ scores.

\section{Baseline Characteristics of Acute Ischemic Stroke Patients in Different Monocyte-to-Lymphocyte Ratio Tertiles}

Demographic, clinical, and laboratory variables, according to MLR tertiles, are depicted in Table 2. The incidence of SAP was significantly higher in the third MLR tertile than the first and second MLR tertiles ( 4 vs. 6.5 and $21.7 \%$, respectively; $P<0.001$; Figure 2). As shown in Table 2, patients with higher MLR levels were older; were more likely to be male, smoker, and diabetic; had higher NIHSS scores and $\mathrm{A}^{2} \mathrm{DS}^{2}$ scores at admission; and had higher leukocyte, monocyte, and PLR counts, as well as lower lymphocyte counts.

\section{Association Between Monocyte-to-Lymphocyte Ratio Levels and Stroke-Associated Pneumonia}

In the univariate logistic regression, continuous MLR values was associated with SAP (odds ratio $[\mathrm{OR}]=1.487,95 \%$ confidence interval $[\mathrm{CI}]=1.359-1.627, P<0.001)$. In the multivariate logistic regression, the occurrence of SAP was used as a dependent variable, and the first MLR tertile was used as a reference for all AIS patients. Multivariate regression analysis showed that the third MLR tertile was an independent variable for predicting the occurrence of SAP $(\mathrm{OR}=6.624,95 \% \mathrm{CI}=3.582-12.250, P<0.001$; Table 3$)$. These differences remained significant after adjusting for confounding and risk factors (Model 1: $\mathrm{OR}=5.156,95 \% \mathrm{CI}=2.207-12.042$, $P<0.001 ;$ Model 2: $\mathrm{OR}=3.503,95 \% \mathrm{CI}=1.066-11.515$ $P=0.039$; Table 3).

ROC analysis showed that the optimal MLR cutoff score for SAP was 0.49 with 0.756 (95\%CI $=0.727-0.782)$ AUROC, $56.73 \%$ sensitivity, $86.57 \%$ specificity, $33.7 \%$ positive predicted value, and $94.2 \%$ negative predicted value (Table 4 ,

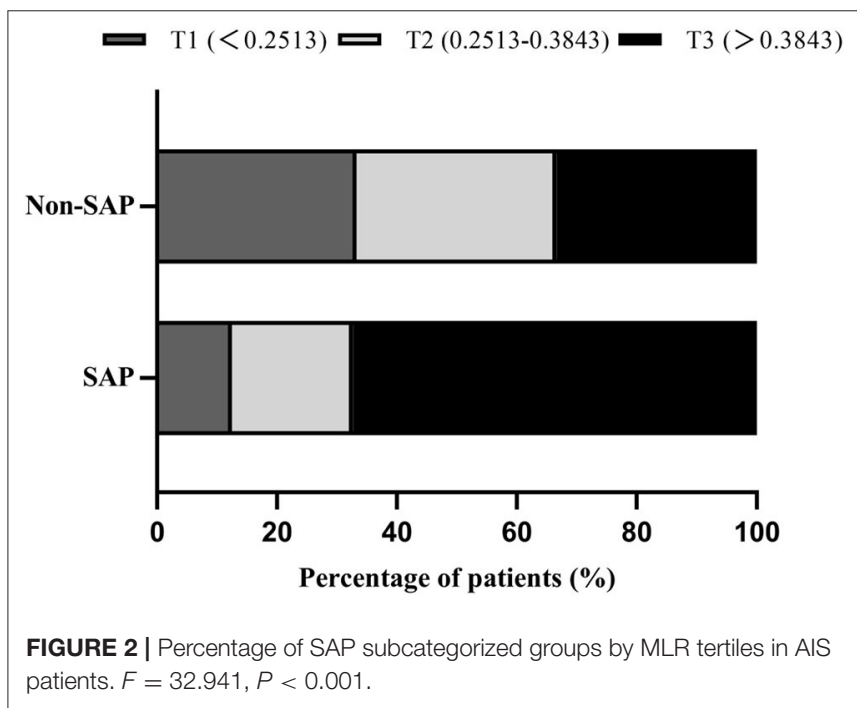


Figure 3). Compared with other biomarkers, the predictive value of MLR was higher than PLR (AUROC $=0.637$, $95 \% \mathrm{CI}=0.606-0.667 ; \quad$ Table 4$)$, leukocyte counts
(AUROC $=0.681,95 \% \mathrm{CI}=0.651-0.711 ;$ Table 4), monocyte counts (AUROC $=0.673,95 \% \mathrm{CI}=0.643-0.703$; Table 4), and lymphocyte counts $(\mathrm{AUROC}=0.686,95 \% \mathrm{CI}=0.656-0.715$;

TABLE 3 | Multivariate logistic regression analysis of clinical determinants of SAP in AIS patients.

\begin{tabular}{|c|c|c|c|c|c|c|}
\hline & \multicolumn{2}{|c|}{ Unadjusted } & \multicolumn{2}{|c|}{ Model 1} & \multicolumn{2}{|c|}{ Model 2} \\
\hline & OR (95\%Cl) & $P$-value & OR $(95 \% \mathrm{Cl})$ & $P$-value & OR $(95 \% \mathrm{Cl})$ & $P$-value \\
\hline \multicolumn{7}{|c|}{ MLR tertiles } \\
\hline Tertile 1 & Reference & & Reference & & Reference & \\
\hline Tertile 2 & $1.658(0.816-3.371)$ & 0.162 & 1.438 (0.564-3.668) & 0.447 & $1.643(0.463-5.836)$ & 0.443 \\
\hline Tertile 3 & $6.624(3.582-12.250)$ & $<0.001$ & $5.156(2.207-12.042)$ & $<0.001$ & $3.503(1.066-11.515)$ & 0.039 \\
\hline
\end{tabular}

MLR, monocyte-to-lymphocyte ratio; AF, atrial fibrillation; NIHSS, National Institute of Health Stroke Scale; GFR, glomerular filtration rate. Model 1: adjusted for age, sex, current smoking, hypertension, diabetes, and AF. Model 2: adjusted for covariates from Model 1 and further adjusted for NIHSS on admission, dysphagia, use of nasogastric tubes, and GFR.

TABLE 4 | Results of ROC analysis about biomarkers with SAP.

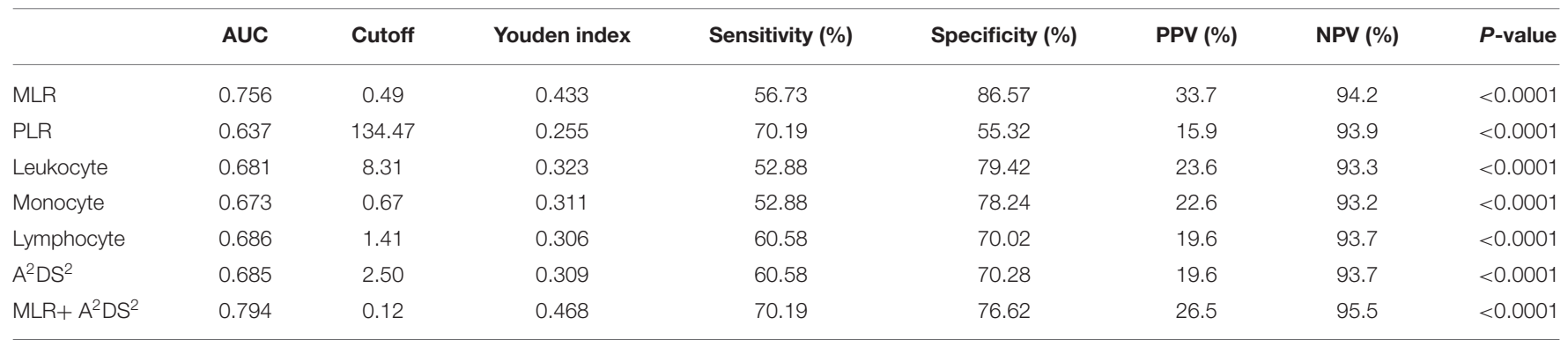

MLR, monocyte-to-lymphocyte ratio; PLR, platelet-to-lymphocyte ratio.

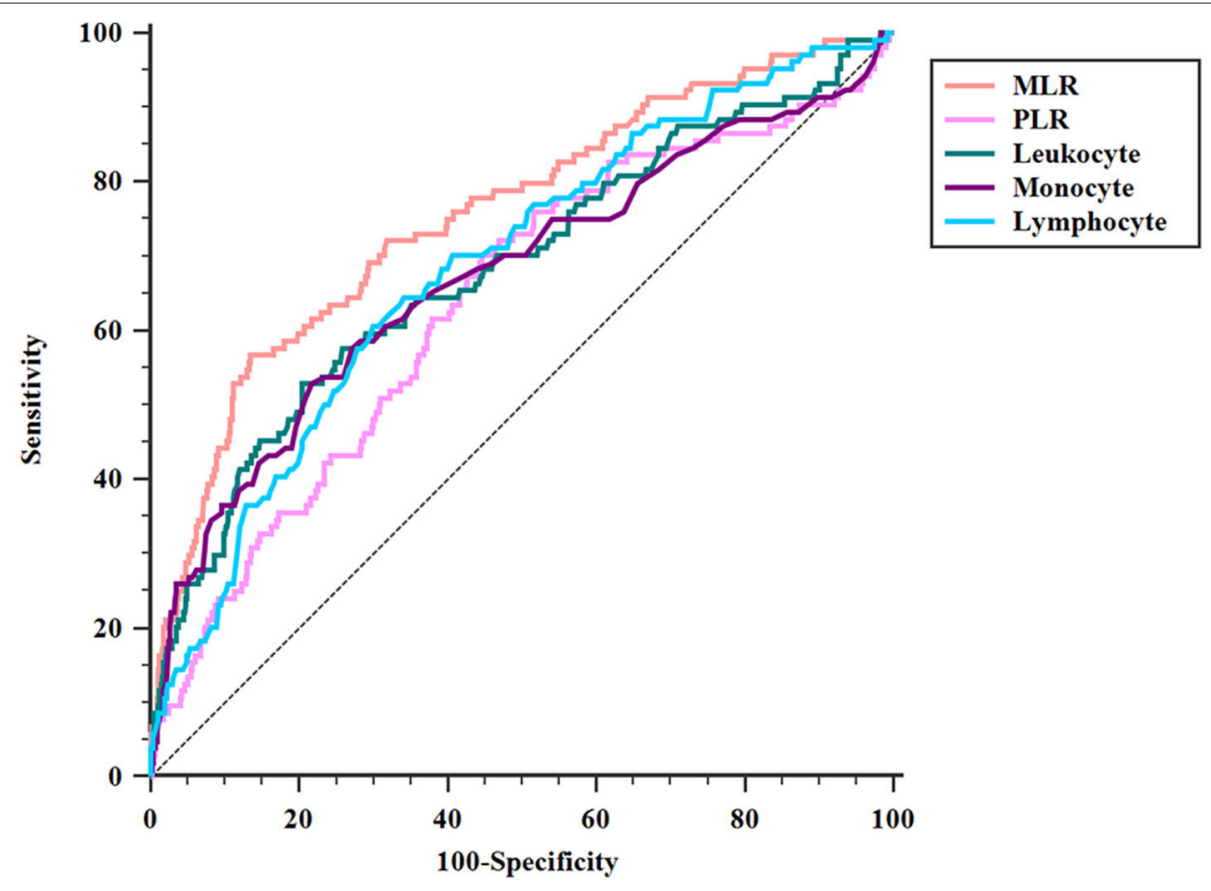

FIGURE 3 | Comparison of area under the receiver operating characteristic curve (AUROC) values among MLR and biomarkers of SAP. MLR vs. PLR, $P<0.001$; MLR vs. leukocyte, $P=0.017$; MLR vs. monocyte, $P<0.001 ;$ MLR vs. lymphocyte, $P=0.012$. 
Table 4). All differences remained significant when MLR was compared with other biomarkers (Figure 3). The AUROC of $\mathrm{A}^{2} \mathrm{DS}^{2}$ score was $0.685(95 \% \mathrm{CI}=0.656-0.715$; Table 4, Figure 4), whereas the AUROC of the modified $\mathrm{A}^{2} \mathrm{DS}^{2}$ score, which combined $\mathrm{A}^{2} \mathrm{DS}^{2}$ score with MLR, was 0.794 (95\%CI $=0.767-0.819 ;$ Table 4, Figure 4). The difference between the modified $\mathrm{A}^{2} \mathrm{DS}^{2}$ scores and MLR $(P=0.032$, Figure 4) was statistically significant, whereas the difference between the modified $\mathrm{A}^{2} \mathrm{DS}^{2}$ score and $\mathrm{A}^{2} \mathrm{DS}^{2}$ score $(P<0.001$, Figure 4) was also statistically significant.

\section{DISCUSSION}

To our knowledge, ours was the first study to explore the relationship between MLR and SAP. We found that higher MLR was significantly associated with the prevalence of SAP in AIS patients. Additionally, we demonstrated that MLR combined with the $\mathrm{A}^{2} \mathrm{DS}^{2}$ model could effectively predict the incidence of SAP. MLR would be an economical, objective, simple blood biomarker to help clinicians recognize AIS patients with a high risk of SAP at an early stage as well as reduce the financial and caring burden of patients. Therefore, higher MLR could be a helpful and valid biomarker for predicting and evaluating SAP in clinical practice.

Compared with the current literature, our study firstly described the relationship between MLR and SAP and compared the discriminating power of SAP in different biomarkers and the modified $\mathrm{A}^{2} \mathrm{DS}^{2}$ score. In our study, 104 (10.7\%) patients were diagnosed with SAP, which was consistent with the previous studies (1-3). We found that AIS patients with older age, higher stroke severity, and dysphagia were more likely to acquire SAP, which was also consistent with the previous studies (5). Furthermore, our study added the treatments, including mechanical ventilation and nasogastric tubes, into the analysis and found that AIS patients who used nasogastric tubes were more likely to acquire SAP. Although nasogastric tubes were used to prevent aspiration in AIS patients, several studies described that prolonged use of nasogastric tube was related to an increased incidence of SAP $(35,36)$. Researchers explained that nasogastric tubes might lead to the dysfunction of the esophageal sphincters and desensitization of the pharyngoglottal adduction reflex (36). Also, we found that SAP patients had lower levels of GFR compared with non-SAP patients, which had not been studied yet.

MLR has been demonstrated as a novel hematological parameter in several medical fields. The elevated MLR level may be a result of the increased monocyte counts or/and decreased lymphocyte counts. In the present study, the MLR levels of SAP patients were higher than non-SAP patients $(0.53$ vs. 0.30 , $P<0.001$; Table 1), and the MLR level of all AIS patients was higher than the non-stroke control patients in a previous study of axial spondyloarthritis ( 0.31 vs. 0.20 , respectively; Table 1) (24). This could be attributed to the inflammatory response led by the occurrence of stroke. Immune cells would infiltrate into the infarction area and then trigger the accumulation and production of chemokines and inflammatory cytokines $(37,38)$; however, the association between MLR and AIS requires further study.

The relationship between MLR and SAP has not been fully studied; however, there are potential mechanisms, such as inflammatory recruitment and immunological suppression after AIS. Monocyte is important in the initiation of inflammatory
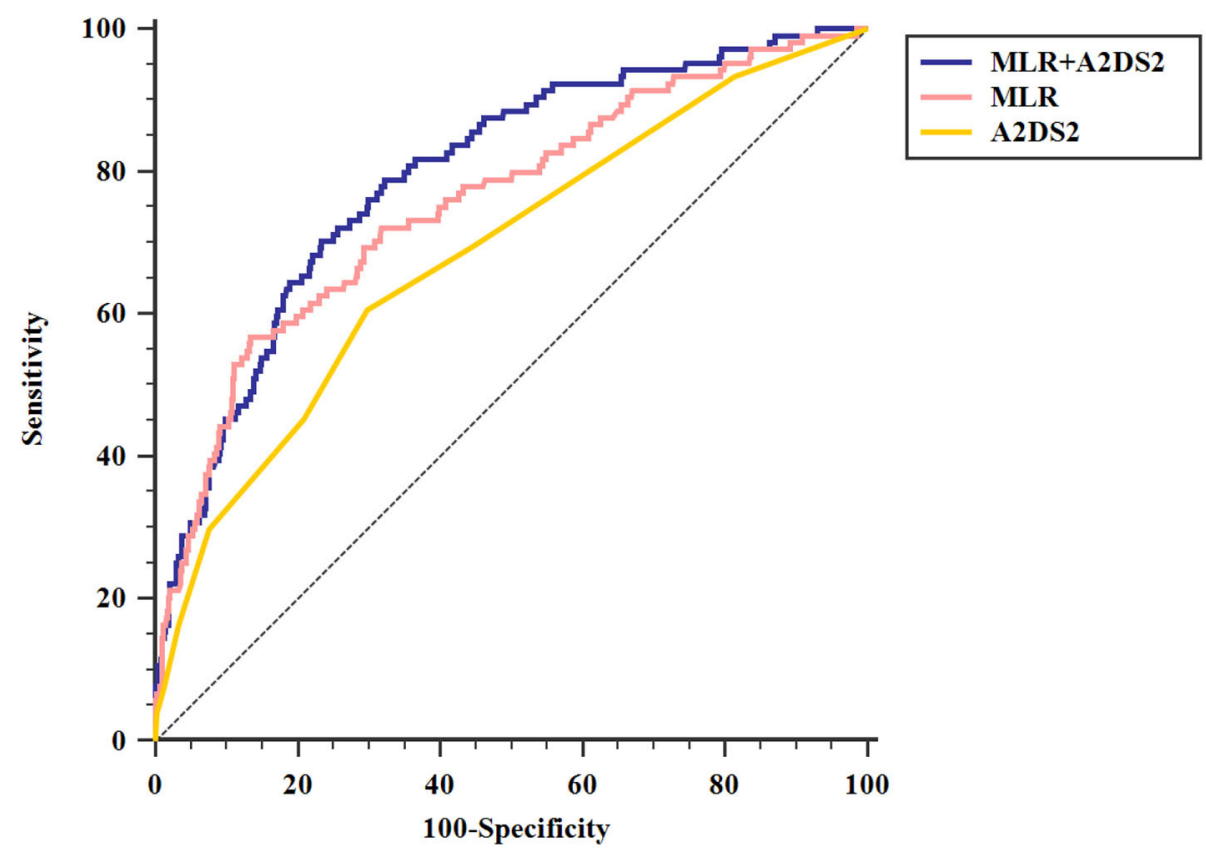

FIGURE 4 | Comparison of area under the receiver operating characteristic curve (AUROC) values among A2DS2 score and MLR combined with A2DS2. MLR+ A2DS2 vs. MLR, $P=0.032 ; \mathrm{MLR}+\mathrm{A} 2 \mathrm{DS} 2$ vs. A2DS2, $P<0.001$ 
processes and is a sentinel and effector of infection (39). During inflammation, monocytes increase and recruit to the site of inflammation, change into terminally differentiated cells, and promote the renewal of dendritic cells and tissue macrophages $(39,40)$. In a previous study, MLR was confirmed to be a predictive factor in the diagnosis of Klebsiella pneumoniae infection (41). Another study performed by Huang et al. (23) showed that higher MLR was significantly associated with community-acquired pneumonia. Thus, increased MLR levels may suggest the initiation of inflammatory processes, which is congruent with previous reports.

Stroke-induced immunodepression syndrome (SIDS) is an immunological change in patients after stroke, and growing evidence has suggested that SIDS could protect our body from secondary inflammatory injury after stroke but would increase the susceptibility to SAP in AIS patients $(42,43)$. When a stroke occurs, brain vessels would be blocked, which subsequently results in hypoxia, nutrient deficiency, and metabolic product accumulation in the infarction area. Thus, a stroke could lead to local inflammatory response and result in SIDS (44). Xabier et al. (45) found that patients with stroke-associated infection had higher monocyte counts. Additionally, Hoffmann et al. (44) found that reduced monocytic human leukocyte antigenDR (HLA-DR), led by SIDS, could predict SAP independently. During the novel coronavirus 2019 pandemic, a new study found that decrease depression of monocytic HLA-DR in critically ill patients may lead to immunosuppression (46). Thus, we hypothesized that SIDS would decrease the expression of monocytic HLA-DR and compensatively increase monocyte counts. Meanwhile, SIDS could result in a rapid decrease of peripheral blood lymphocyte counts and functional inactivation of T cells (47). Konstantin et al. (48) found that lymphocytes suffered a comprehensive apoptotic loss after stroke, and a catecholamine-mediated lymphocytic defect was essential for stroke-associated infection. Meanwhile, Park et al. (25) found that lower LMR on day 7 is associated with worse outcomes at 3 months in AIS patients and suggested that LMR may be a helpful biomarker for indicating SIDS for the features of SIDS are decreased of lymphocyte counts and deactivation of monocytes. Our study found that high MLR at admission was associated with the occurrence of SAP, which added and supported their hypothesis of SIDS marker in the acute phase of AIS. Therefore, SIDS could influence the monocyte and lymphocyte counts, which could explain the association between MLR and SAP.

In our study, we also made comparisons between MLR and several cell counts, PLR and $\mathrm{A}^{2} \mathrm{DS}^{2}$ model. MLR and PLR were reported to be independent risk factors in many infectious diseases (49). Also, the counts of leukocytes, monocytes, and lymphocytes could directly reflect the inflammatory and immunity process. Among these biomarkers, MLR was the best biomarker for predicting SAP in our study. Stroke, as major stress for the human body, would trigger reactions to this stressful event such as inflammatory and immune responses after onset. However, there were a small number of researches that studied the associations between SAP and easily obtained biomarkers of inflammatory and immunity and compared their predictive values. Our study filled these gaps in previous studies and found that MLR was an economical, objective, and simple parameter to predict SAP in an early stage. Hoffmann et al. (14) developed the $\mathrm{A}^{2} \mathrm{DS}^{2}$ scoring system to predict the risk of SAP, which has validated in different cohorts $(3,14,15)$. In a valid external comparison, Zapata-Arriaza et al. (15) found that $\mathrm{A}^{2} \mathrm{DS}^{2}$ had the highest sensitivity (87\%) and specificity (92.8\%), compared with the AIS-associated pneumonia score and the pre-stroke Independence, Sex, Age, National Institutes of Health Stroke Scale. Our current study described that the SAP patients had a higher $\mathrm{A}^{2} \mathrm{DS}^{2}$ score than non-SAP patients, which was consistent with the prediction model. Also, we found that the modified $\mathrm{A}^{2} \mathrm{DS}^{2}$ score, which combined the $\mathrm{A}^{2} \mathrm{DS}^{2}$ score with MLR, had the highest discriminating power of SAP, compared with the $\mathrm{A}^{2} \mathrm{DS}^{2}$ score $(P<0.001$, Figure 4$)$ or $\operatorname{MLR}(P=0.032$, Figure 4). The AUROC of the modified $\mathrm{A}^{2} \mathrm{DS}^{2}$ score (0.794, Table 4) was also higher than the $\mathrm{A}^{2} \mathrm{DS}^{2}$ score (0.685, Table 4) and MLR (0.756, Table 4). As the indexes of the $\mathrm{A}^{2} \mathrm{DS}^{2}$ score only included demographic and clinical parameters at admission, it was developed for use on the first day after stroke onset. Thus, it cannot reflect the inflammatory process in time. Although MLR could reflect the inflammatory response in AIS patients, it could also modify the $\mathrm{A}^{2} \mathrm{DS}^{2}$ score.

There are some limitations to our current study. First, this was a single-center and retrospective study; therefore, we cannot establish causality between MLR and SAP, necessitating the use of future multicenter, prospective studies. Secondly, MLR was only measured at admission and unable to be measured at other time points (beyond $24 \mathrm{~h}$ ), so we cannot evaluate the association between MLR and SAP dynamically. In further studies, we would measure MLR at multiple times during the hospitalization to investigate whether a high MLR is developing only after stroke in certain patients or is this an interindividual difference that patients already present with independent of the stroke. Thirdly, the newest inflammatory factors, such as interleukin-6, were not included in this study. Further studies could record more inflammatory factors to clearly explain the relationship between inflammation and pneumonia.

In conclusion, the present study showed that higher MLR was significantly associated with SAP in AIS patients. When combined with $\mathrm{A}^{2} \mathrm{DS}^{2}$ scores, the newly developed model had a higher discriminating power of SAP. MLR is beneficial for clinicians to recognize patients with a high risk of SAP at an early stage and is an effective way to improve clinical care of SAP patients. Therefore, higher MLR could be a helpful and valid biomarker for predicting SAP in clinical practice.

\section{DATA AVAILABILITY STATEMENT}

The data are not publicly available due to privacy or ethical restrictions. Requests to access these datasets should be directed to Zhen Wang, wangzhen@wzhospital.cn.

\section{ETHICS STATEMENT}

The studies involving human participants were reviewed and approved by the Ethics Committee of First Affiliated Hospital of Wenzhou Medical University. Written informed consent for 
participation was not required for this study in accordance with the national legislation and the institutional requirements.

\section{AUTHOR CONTRIBUTIONS}

H-RC: conceptualization, data curation, formal analysis, and writing the original draft. J-YS, Y-NZ, and Y-BC: data curation and formal analysis. G-QL: data curation. G-QH: conceptualization and project administration. J-CH: conceptualization, resources, and writing-review and editing. ZW: funding acquisition, resources, and writing-review, and editing. All authors contributed to the article and approved the submitted version.

\section{REFERENCES}

1. Badve MS, Zhou Z, van de Beek D, Anderson CS. Frequency of post-stroke pneumonia: systematic review and meta-analysis of observational studies. Int J Stroke. (2019) 14:125-36. doi: 10.1177/1747493018806196

2. Ingeman A, Andersen G, Hundborg HH, Svendsen ML, Johnsen SP. In-hospital medical complications, length of stay, and mortality among stroke unit patients. Stroke. (2011) 42:3214-8. doi: 10.1161/STROKEAHA.110.610881

3. Smith CJ, Bray BD, Hoffman A, Meisel A, Heuschmann PU, Wolfe CD, et al. Can a novel clinical risk score improve pneumonia prediction in acute stroke care? A UK multicenter cohort study. J Am Heart Assoc. (2015) 4:e001307. doi: 10.1161/JAHA.114.001307

4. Koennecke HC, Belz W, Berfelde D, Endres M, Fitzek S, Hamilton $\mathrm{F}$, et al. Factors influencing in-hospital mortality and morbidity in patients treated on a stroke unit. Neurology. (2011) 77:965-72. doi: 10.1212/WNL.0b013e31822dc795

5. Finlayson O, Kapral M, Hall R, Asllani E, Selchen D, Saposnik G. Risk factors, inpatient care, and outcomes of pneumonia after ischemic stroke. Neurology. (2011) 77:1338-45. doi: 10.1212/WNL.0b013e31823152b1

6. Zhang S, He WB, Chen NH. Causes of death among persons who survive an acute ischemic stroke. Curr Neurol Neurosci Rep. (2014) 14:467. doi: 10.1007/s11910-014-0467-3

7. Saposnik G, Hill MD, O’Donnell M, Fang J, Hachinski V, Kapral MK. Variables associated with 7-day, 30-day, and 1-year fatality after ischemic stroke. Stroke. (2008) 39:2318-24. doi: 10.1161/STROKEAHA.107.510362

8. Schwarz S. Prophylactic antibiotic therapy for preventing poststroke infection. Neurotherapeutics. (2016) 13:783-90. doi: 10.1007/s13311-016-0466-y

9. Vermeij JD, Westendorp WF, Dippel DW, van de Beek D, Nederkoorn PJ. Antibiotic therapy for preventing infections in people with acute stroke. Cochrane Database Syst Rev. (2018) 1:Cd008530. doi: 10.1002/14651858.CD008530.pub3

10. Ding Y, Yan Y, Niu J, Zhang Y, Gu Z, Tang P, et al. Braden scale for assessing pneumonia after acute ischaemic stroke. BMC Geriatr. (2019) 19:259. doi: 10.1186/s12877-019-1269-x

11. Harms H, Grittner U, Droge H, Meisel A. Predicting post-stroke pneumonia: the PANTHERIS score. Acta Neurol Scand. (2013) 128:178-84. doi: 10.1111/ane.12095

12. Kalra L, Smith CJ. Elevated C-reactive protein increases diagnostic accuracy of algorithm-defined stroke-associated pneumonia in afebrile patients. Int J Stroke. (2019) 14:167-73. doi: 10.1177/1747493018798527

13. Vart P, Bettencourt-Silva JH, Metcalf AK, Bowles KM, Potter JF, Myint PK. Low estimated glomerular filtration rate and pneumonia in stroke patients: findings from a prospective stroke registry in the East of England. Clin Epidemiol. (2018) 10:887-96. doi: 10.2147/CLEP.S156176

14. Hoffmann S, Malzahn U, Harms H, Koennecke HC, Berger K, Kalic M, et al. Development of a clinical score (A2DS2) to predict pneumonia in acute ischemic stroke. Stroke. (2012) 43:2617-23. doi: 10.1161/STROKEAHA.112.653055

\section{FUNDING}

This work was supported by the Projects of Provincial Natural Science Foundation of Zhejiang (no. LY19H090013) and the Projects of Medical Health Science and Technology of Zhejiang (no. 2020KY637).

\section{ACKNOWLEDGMENTS}

We thank the study participants and the clinical staff at all participating hospitals for their support and contribution to this project.

15. Zapata-Arriaza E, Moniche F, Blanca PG, Bustamante A, EscuderoMartinez I, Ucles $\mathrm{O}$, et al. External validation of the ISAN, A2DS2, and AIS-APS scores for predicting stroke-associated Pneumonia. J Stroke Cerebrovasc Dis. (2018) 27:673-6. doi: 10.1016/j.jstrokecerebrovasdis.2017. 09.059

16. Li X, Wu M, Sun C, Zhao Z, Wang F, Zheng X, et al. Using machine learning to predict stroke-associated pneumonia in Chinese acute ischaemic stroke patients. European J Neurol. (2020) 27:1656-63. doi: 10.1111/ene.14295

17. Huang G, Cheng H, Wu Y, Cheng Q, Wang Y, Fu J, et al. Reduced Vitamin D levels are associated with stroke-associated pneumonia in patients with acute ischemic stroke. Clin Interv Aging. (2019) 14:2305-14. doi: $10.2147 /$ CIA.S230255

18. Zapata-Arriaza E, Mancha F, Bustamante A, Moniche F, Pardo-Galiana B, Serrano-Gotarredona $\mathrm{P}$, et al. Biomarkers predictive value for early diagnosis of Stroke-Associated Pneumonia. Ann Clin Transl Neurol. (2019) 6:1882-7. doi: 10.1002/acn3.50849

19. Jin R, Liu S, Wang M, Zhong W, Li G. Inhibition of CD147 attenuates strokeassociated pneumonia through modulating lung immune response in mice. Front Neurol. (2019) 10:853. doi: 10.3389/fneur.2019.00853

20. Nam KW, Kim TJ, Lee JS, Kwon HM, Lee YS, Ko SB, et al. High neutrophilto-lymphocyte ratio predicts stroke-associated pneumonia. Stroke. (2018) 49:1886-92. doi: 10.1161/STROKEAHA.118.021228

21. Kwan J, Horsfield G, Bryant T, Gawne-Cain M, Durward G, Byrne CD, et al. IL-6 is a predictive biomarker for stroke associated infection and future mortality in the elderly after an ischemic stroke. Exp Gerontol. (2013) 48:960-5. doi: 10.1016/j.exger.2013.07.003

22. Jiang Y, Zang M, Li S. Serum PLR and LMR in Behçet's disease: can they show the disease activity? Medicine. (2017) 96:e6981. doi: 10.1097/MD.0000000000006981

23. Huang Y, Liu A, Liang L, Jiang J, Luo H, Deng W, et al. Diagnostic value of blood parameters for community-acquired pneumonia. Int Immunopharmacol. (2018) 64:10-5. doi: 10.1016/j.intimp.2018.08.022

24. Huang Y, Deng W, Zheng S, Feng F, Huang Z, Huang Q, et al. Relationship between monocytes to lymphocytes ratio and axial spondyloarthritis. Int Immunopharmacol. (2018) 57:43-6. doi: 10.1016/j.intimp.2018.02.008

25. Park MG, Kim MK, Chae SH, Kim HK, Han J, Park KP. Lymphocyte-tomonocyte ratio on day 7 is associated with outcomes in acute ischemic stroke. Neurol Sci. (2018) 39:243-9. doi: 10.1007/s10072-017-3163-7

26. Yu JI, Park HC, Yoo GS, Paik SW, Choi MS, Kim HS, et al. Clinical significance of systemic inflammation markers in newly diagnosed, previously untreated hepatocellular carcinoma. Cancers. (2020) 12:1300. doi: $10.3390 /$ cancers 12051300

27. van der Willik KD, Koppelmans V, Hauptmann M, Compter A, Ikram MA, Schagen SB. Inflammation markers and cognitive performance in breast cancer survivors 20 years after completion of chemotherapy: a cohort study. Breast Cancer Res. (2018) 20:135. doi: 10.1186/s13058-018-1062-3

28. Tu WJ, Dong X, Zhao SJ, Yang DG, Chen H. Prognostic value of plasma neuroendocrine biomarkers in patients with acute ischaemic stroke. J Neuroendocrinol. (2013) 25:771-8. doi: 10.1111/jne.12052 
29. Smith CJ, Kishore AK, Vail A, Chamorro A, Garau J, Hopkins SJ, et al. Diagnosis of stroke-associated pneumonia: recommendations from the pneumonia in stroke consensus group. Stroke. (2015) 46:2335-40. doi: 10.1161/STROKEAHA.115.009617

30. Huang GQ, Lin YT, Wu YM, Cheng QQ, Cheng HR, Wang Z. Individualized prediction of stroke-associated pneumonia for patients with acute ischemic stroke. Clin Interv Aging. (2019) 14:1951-62. doi: 10.2147/CIA.S225039

31. Brodsky MB, Suiter DM, González-Fernández M, Michtalik HJ, Frymark TB, Venediktov R, et al. Screening accuracy for aspiration using bedside water swallow tests: a systematic review and meta-analysis. Chest. (2016) 150:148-63. doi: 10.1016/j.chest.2016.03.059

32. Japan TO-R-LSo. The Treatment Guideline of Deglutition Disorders, 2nd Edn. Kanehara Shuppan (2012).

33. Zhang J, Song Y, Zhang C, Zhi X, Fu H, Ma Y, et al. Circulating MiR-16-5p and MiR-19b-3p as two novel potential biomarkers to indicate progression of gastric cancer. Theranostics. (2015) 5:733-45. doi: 10.7150/thno.10305

34. Ay H, Gungor L, Arsava E, Rosand J, Vangel M, Benner T, et al. A score to predict early risk of recurrence after ischemic stroke. Neurology. (2010) 74:128-35. doi: 10.1212/WNL.0b013e3181ca9cff

35. Matz K, Seyfang L, Dachenhausen A, Teuschl Y, Tuomilehto J, Brainin $\mathrm{M}$, et al. Post-stroke pneumonia at the stroke unit - a registry based analysis of contributing and protective factors. BMC Neurol. (2016) 16:107. doi: 10.1186/s12883-016-0627-y

36. Gomes GF, Pisani JC, Macedo ED, Campos AC. The nasogastric feeding tube as a risk factor for aspiration and aspiration pneumonia. Curr Opin Clin Nutr Metab Care. (2003) 6:327-33. doi: 10.1097/01.mco.0000068970.34812.8b

37. Nakamura A, Otani K, Shichita T. Lipid mediators and sterile inflammation in ischemic stroke. Int Immunol. (2020). doi: 10.1093/intimm/dxaa027. [Epub ahead of print].

38. Shichita T, Sakaguchi R, Suzuki M, Yoshimura A. Post-ischemic inflammation in the brain. Front Immunol. (2012) 3:132. doi: 10.3389/fimmu.2012. 00132

39. Bieber K, Autenrieth SE. Insights how monocytes and dendritic cells contribute and regulate immune defense against microbial pathogens. Immunobiology. (2015) 220:215-26. doi: 10.1016/j.imbio.2014.10.025

40. Tsuchimoto Y, Asai A. M2b monocytes provoke bacterial pneumonia and gut bacteria-associated sepsis in alcoholics. J Immunol. (2015) 195:5169-77. doi: 10.4049/jimmunol.1501369

41. Wang JL, Lu XY, Xu XH, Zhang KJ, Gong H, Lv D, et al. Predictive role of monocyte-to-lymphocyte ratio in patients with Klebsiella pneumonia infection: a single-center experience. Medicine. (2019) 98:e17215. doi: 10.1097/MD.0000000000017215
42. Wang R, Zeng J, Wang F, Zhuang X, Chen X, Miao J. Risk factors of hemorrhagic transformation after intravenous thrombolysis with rt-PA in acute cerebral infarction. QJM. (2019) 112:323-6. doi: 10.1093/qjmed/hcy292

43. Liu DD, Chu SF, Chen C, Yang PF, Chen NH, He X. Research progress in stroke-induced immunodepression syndrome (SIDS) and stroke-associated pneumonia (SAP). Neurochem Int. (2018) 114:42-54. doi: 10.1016/j.neuint.2018.01.002

44. Hoffmann S, Harms H, Ulm L, Nabavi DG, Mackert BM, Schmehl I, et al. Stroke-induced immunodepression and dysphagia independently predict stroke-associated pneumonia - the PREDICT study. J Cereb Blood Flow Metab. (2017) 37:3671-82. doi: 10.1177/0271678X16671964

45. Urra X, Cervera A, Obach V, Climent N, Planas AM, Chamorro A. Monocytes are major players in the prognosis and risk of infection after acute stroke. Stroke. (2009) 40:1262-8. doi: 10.1161/STROKEAHA.108.532085

46. Spinetti T, Hirzel C, Fux M, Walti L, Schober P, Stueber F, et al. Reduced monocytic human leukocyte antigen-DR expression indicates immunosuppression in critically Ill COVID-19 patients. Anesth Analg. (2020) 131:993-9. doi: 10.1213/ane.0000000000005044

47. Klehmet J, Harms H, Richter M, Prass K, Volk HD, Dirnagl U, et al. Strokeinduced immunodepression and post-stroke infections: lessons from the preventive antibacterial therapy in stroke trial. Neuroscience. (2009) 158:118493. doi: 10.1016/j.neuroscience.2008.07.044

48. Prass K, Meisel C, Höflich C, Braun J, Halle E, Wolf T, et al. Strokeinduced immunodeficiency promotes spontaneous bacterial infections and is mediated by sympathetic activation reversal by poststroke $\mathrm{T}$ helper cell type 1-like immunostimulation. J Exp Med. (2003) 198:725-36. doi: 10.1084/jem.20021098

49. Yang AP, Liu JP, Tao WQ, Li HM. The diagnostic and predictive role of NLR, d-NLR and PLR in COVID-19 patients. Int Immunopharmacol. (2020) 84:106504. doi: 10.1016/j.intimp.2020.106504

Conflict of Interest: The authors declare that the research was conducted in the absence of any commercial or financial relationships that could be construed as a potential conflict of interest.

Copyright (C) 2020 Cheng, Song, Zhang, Chen, Lin, Huang, He and Wang. This is an open-access article distributed under the terms of the Creative Commons Attribution License (CC BY). The use, distribution or reproduction in other forums is permitted, provided the original author(s) and the copyright owner(s) are credited and that the original publication in this journal is cited, in accordance with accepted academic practice. No use, distribution or reproduction is permitted which does not comply with these terms. 